\title{
Synthesis and characterization of in situ prepared poly (methyl methacrylate) nanocomposites ${ }^{\dagger}$
}

\author{
SHAHZADA AHMAD, SHARIF AHMAD ${ }^{\dagger \dagger}$ and S A AGNIHOTRY* \\ Electronic Materials Division, National Physical Laboratory, New Delhi 110 012, India \\ ${ }^{\dagger \dagger}$ Department of Chemistry, Jamia Millia Islamia, New Delhi 110 025, India
}

MS received 8 August 2006; revised 28 December 2006

\begin{abstract}
Hybrid materials, which consist of organic-inorganic materials, are of profound interest owing to their unexpected synergistically derived properties. These hybrid materials replaced the pristine polymers due to their higher strength and stiffness in the recent years. In the present work, studies concerning the preparation of poly (methyl methacrylate) (PMMA), $\mathrm{PMMA}_{\mathrm{S}} \mathrm{SiO}_{2}$, and $\mathrm{PMMA} / \mathrm{TiO}_{2}$ nanocomposites are reported. These nanocomposite polymers were synthesized by means of free radical polymerization of methyl methacrylate using benzoyl peroxide as an initiator in a water medium. Further 'sol-gel' transformation based hydrolysis and condensation of $\mathrm{Ti}$ and $\mathrm{Si}$ alkoxides were used to prepare the inorganic phase during the polymerization process of MMA.
\end{abstract}

Keywords. PMMA; polymer nanocomposite; sol-gel; SEM; FTIR.

\section{Introduction}

Polymers are of profound interest to society and are replacing metals in diverse fields of life, which can be further modified according to modern application. Organicinorganic hybrid materials are hi-tech because they can present simultaneously both the properties of an inorganic molecule besides the usual properties of polymer (an organic molecule). These hybrid materials sometimes lead to unexpected new properties, which are often not exhibited by individual compounds and thus open a new avenue for chemists, physicists and materials scientists. These hybrid materials are new, versatile class of materials, exhibiting a vast application potential, due to their tailorable mechanical, optical and electrical properties (Meneghetti and Qutubuddin 2004). Hybrid materials with polymers as organic constituent are often called 'polymer composites'. They offer improved properties including higher strength and stiffness than pristine polymers (Mark 1996). Different types of organic constituents or monomers can be used and various methods can be adopted according to requirements. 'Nanocomposite polymers' are polymers, which have dispersed phase in them with ultra-fine dimension typically of some nanometer (Qutubuddin and Fu 2002). The choice of the polymer and the dispersed phase is determined by the properties required for the end product.

\footnotetext{
*Author for correspondence (agni@ mail.nplindia.ernet.in) 'Paper presented at the Indo-Singapore symposium on 'Advanced Functional Materials', IIT Mumbai, 2006.
}

These nanocomposites exhibit enhanced thermal stability with greater dimensional stability, stiffness, strength, low thermal expansion and fire retardancy and also unusual magnetic, optical and electronic properties than their counterpart (Qiang et al 2004; Wang et al 2005). Nano-scale composite materials containing titanium oxides are interesting because of their potential applications in optoelectronic devices (Suzuki et al 2002). A great deal of research has been focused on both synthesis of high quality, transparent films consisting of polymer- $\mathrm{TiO}_{2}$ hybrid nanocomposites, and their linear optical properties (Lee and Chen 2001). Recently, nonlinear-optical properties of such materials have also received attention.

There are three common approaches for the synthesis of nanocomposite polymers, which have been adopted so far (Ahmadi et al 2004). The first approach is the in situ polymerization technique, the second approach is dissolving the polymer in a solvent and then mixing with the dispersing media, while the third approach is of melt intercalation and involves heating the polymer above its glass transition temperature and then mixing it with the dispersing medium. The first approach is rather simple and largely favoured, as it neither requires the costly solvent nor does it consume energy and is very economical too.

Suspension polymerization is a type of free radical polymerization and requires four parts: a non-water soluble monomer, a surfactant, a free-radical initiator, and water. Before polymerization, the components can either be mixed together in preparation for a batch reaction, or kept separately and continuously added to the reactor to have a better control over the reaction rate. In both batch and continuous reactions, 
when the monomer is added to the reactor, it first exists in relatively large droplets, due to its insolubility in water. The initiator generates free radical that attacks the double bond on a monomer molecule, bonding to it and causing the combined molecule to have a free radical at the end. This reacts with another monomer molecule, and the process continues until another initiator free radical terminates the chain. Typically there is one growing chain per micelle at a time, leading to polymer particles with nearly identical molecular weights. When all monomer has been consumed, what remains is a colloidal dispersion of solid polymer particles in water. This motivated us to attempt a new route in which nano scale ceramic particles are precipitated in a polymer host. A simple method, viz. 'sol-gel', was followed for the same (Schmidt 1998; Limmer et al 2002). In this paper, we propose a new method to prepare PMMA nanocomposite by free radical suspension polymerization using water as a medium, with in situ 'sol-gel' transformation. The morphology, structural properties and thermal behaviour of PMMA, PMMA-SiO 2 and PMMA-TiO nanocomposite polymers are reported here.

\section{Experimental}

\subsection{Materials}

Methyl methacrylate (MMA) was made inhibitor free by passing through activated alumina column, ethyl alcohol and hydrochloric acid (37\%), all supplied by Merck, Germany. Tetraethyl orthosilicate (TEOS) and titanium (IV) isopropoxide (Ti-iP) used as precursors for the inorganic fillers were obtained from Aldrich. Benzoyl peroxide (Loba chemicals, India) was used as an initiator while poly vinyl alcohol and anhydrous di-sodium hydrogen phosphate used were respectively from Aldrich and $\mathrm{CDH}$, India.

\subsection{Synthesis of polymer}

A typical procedure to prepare PMMA was followed which involved placing $20 \mathrm{ml}$ of MMA, $0.15 \mathrm{~g}(0.75 \%)$ of BPO in $100 \mathrm{ml}$ of water and $0.5 \mathrm{~g}$ of PVA and $5 \mathrm{~g}$ of di-sodium hydrogen phosphate in a $250 \mathrm{ml}$ three-necked round bottom flask and the solution was stirred for $1 \mathrm{~h}$ at $80^{\circ} \mathrm{C}$ to complete the polymerization of monomer. Nitrogen was bubbled into the flask throughout the reaction. The solution was then filtered, washed and finally dried at $100^{\circ} \mathrm{C}$ for $24 \mathrm{~h}$ under vacuum to yield PMMA solid material.

2.2a Synthesis of PMMA-SiO $\mathrm{P}_{2}$ and $\mathrm{PMMA}-\mathrm{TiO}_{2}$ composites: The same procedure was followed except after half an hour of reaction the sol was added. The sol was prepared by mixing TEOS $(2 \mathrm{ml})$, deionized water $(0.4 \mathrm{ml})$ and $\mathrm{HCl}(0.04 \mathrm{ml}$, used to catalyze the hydrolysis $)$ in a beaker and pre-hydrolyzed in air and then the mixture was sonicated for $15 \mathrm{~min}$ to facilitate the conversion of ethoxy ligands to $\mathrm{Si}-\mathrm{OH}$ groups. The $\mathrm{TiO}_{2}$-based solution was prepared using titanium isopropoxide (Ti-iP), de-ionized water, ethanol and hydrochloric acid, according to the method described elsewhere (Burgos and Langlet 1999). Ti-iP $(2 \mathrm{ml})$ was first mixed with ethanol $(20 \mathrm{ml})$ in a beaker and stirred for $30 \mathrm{~min}$. A mixture of de-ionized water and $\mathrm{HCl}$ was poured under stirring into the transparent solution to promote hydrolysis. The Ti-iP concentration in the solution was fixed at $0.4 \mathrm{M}$ with an under stoichiometric ratio of water to $\mathrm{Ti}-\mathrm{iP}(\mathrm{rw})$ of $0 \cdot 82$. And the $\mathrm{pH}$ value of 1.3 was used to obtain a stable solution (i.e. with longest gelation time). Furthermore, this homogeneous mixture was added dropwise over $30 \mathrm{~min}$ into the reaction media of monomers with rigorous stirring to avoid local inhomogeneities.

\subsection{Instrumentation}

Morphology was studied using scanning electron microscopy, LEO 440, and the samples were gold coated prior to measurements. XRD measurements were carried out on Philips PW3710 diffractometer while thermogravimetric analysis (TGA) was performed on Perkin-Elmer TGA7 in the presence of nitrogen and the samples were heated to $500^{\circ} \mathrm{C}$ at a rate of $20^{\circ} \mathrm{C} / \mathrm{min}$. Infrared spectra of polymer samples were recorded as $\mathrm{KBr}$ pellets, in the region $4000-400 \mathrm{~cm}^{-1}$ on a computer interfaced Perkin-Elmer FX-RX1.

\section{Results and discussion}

\subsection{Morphology}

Figure 1 illustrates the micrographs of the obtained composites revealing that their production was successfully achieved yielding materials with particles well dispersed within the matrices. Figure 1(a) shows the micrograph of virgin polymers and it can be seen that the distribution of size is not uniform and the particles size varies. They range from $3-12 \mu \mathrm{m}$ in size and their chain formation is clearly visible from the micrograph. The virgin polymer also exhibits porous nature while the pores disappear in the composite structure. This illustrates that the nanoparticles are intercalated into the structure of polymer. In figure 1(b), SEM picture shows silica nanoparticles adhered to the polymer surface. The hydrophilic silica particles on the surface of the polymer, hydrophilic due to the hydroxyl groups on the silica surface, combined with inherent surface roughness impart hydrophilic nature, according to Cassie's equation (Nakajima et al 2001). During the reaction, the hydrophilic silica particles migrated to the polymer water interface due to Van der Waal's attraction. The micrograph shows a distribution of two groups of about $1-2 \mu \mathrm{m}$ and $0.5 \mu \mathrm{m}$ silica particles, which are spherical in shape. Figure 1(b) exhibits polymers coated with silica particles but with non-uniform distribution. There are some 

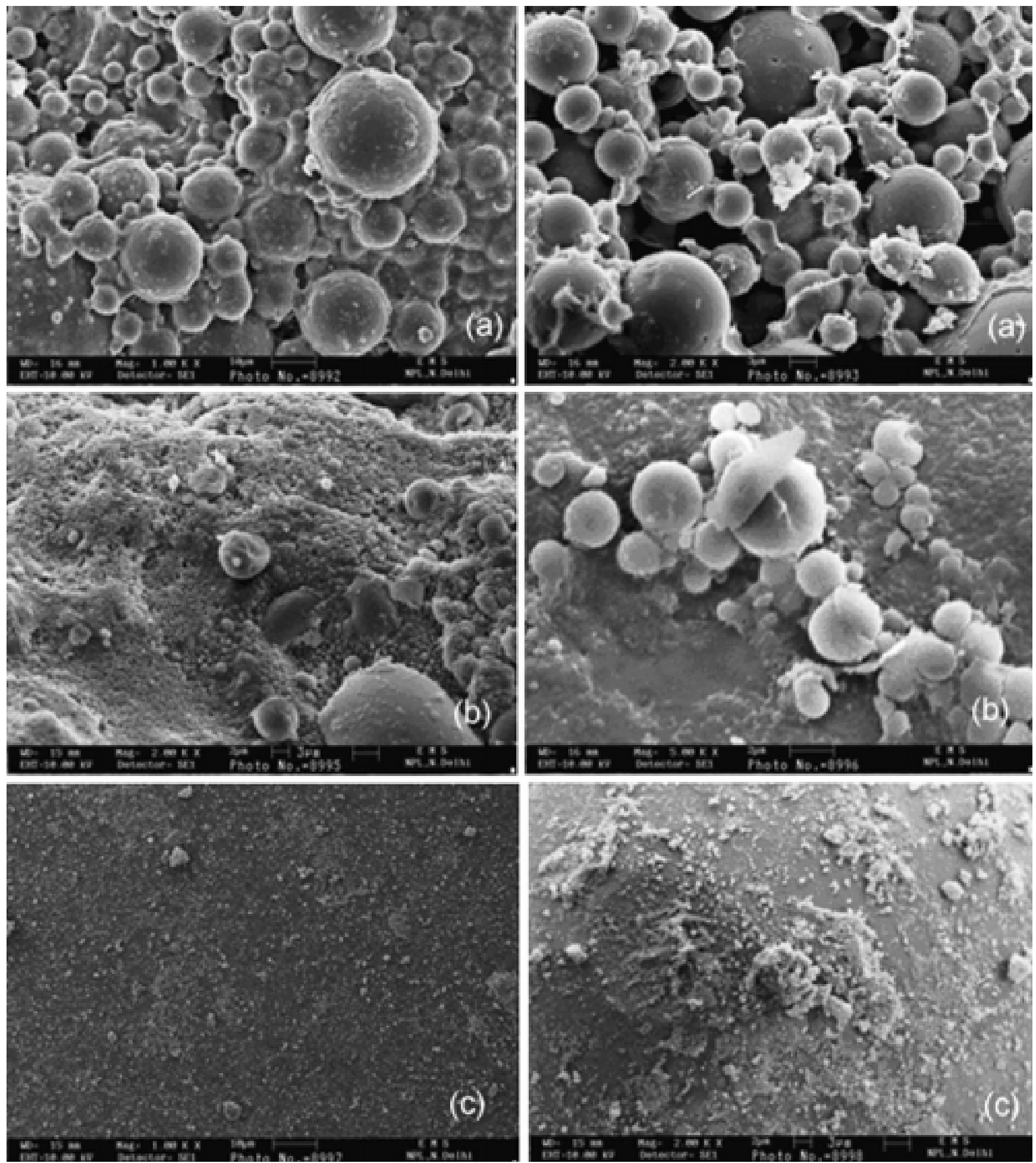

Figure 1. SEM of (a) PMMA, (b) $\mathrm{PMMA}-\mathrm{SiO}_{2}$ and (c) $\mathrm{PMMA}^{-\mathrm{TiO}_{2}}$ composites.

agglomerates also formed which is reflected as larger particles and their size vary from 1-5 $\mu \mathrm{m}$. Figure 1(c) indicates spherical aggregates with diameter $<0.2 \mu \mathrm{m}$. It can be seen that the $\mathrm{TiO}_{2}$ particles are well dispersed on the PMMA matrix and coated the polymer uniformly with an average particle size of $0 \cdot 2 \mu \mathrm{m}$. The distribution size is uniform but at some places it has formed agglomerates.

It can thus be concluded that the planarity of the composite can be significantly improved by using the surface functionalized silica and titania oxide particles. 


\subsection{XRD studies}

Figure 2 illustrates the diffractograms of PMMA and PMMA-SiO $\mathrm{S}_{2}$ composites in the $2 \theta$ range between 5 and 90 degree, which are similar and without any sharp diffraction peaks confirming their non-crystalline nature. The interlayer spacing of the system was determined by the diffraction peak in the $\mathrm{X}$-ray method, using the Bragg equation

$$
\lambda=2 d \sin \theta,
$$

where $d$ is the spacing between diffractional lattice planes, $\theta$ the diffraction position while $\lambda$ the wavelength of the X-ray $(1 \cdot 5405 \AA)$.

PMMA is known to be an amorphous polymer (Meneghetti et al 2004) and shows three broad peaks at $2 \theta$ values of $12^{\circ}, 30^{\circ}$ and $32^{\circ}$ ( $d$ spacing around $7 \AA, 2.94 \AA$ and $2 \cdot 79 \AA$ ), with their intensity decreasing systematically. The shape of the first most intense peak reflects the ordered packing of polymer chains while the second peak denotes the ordering inside the main chains. The addition of $\mathrm{SiO}_{2}$ does not induce any crystallinity in these polymers. This also explains the homogeneous nature of these samples.

\subsection{FTIR}

Figure 3 depicts the FT-IR spectra of PMMA, PMMA$\mathrm{SiO}_{2}$ and $\mathrm{PMMA}-\mathrm{TiO}_{2}$. It is evident that all the three spectra are similar except for a few changes in the spectra of the nanocomposites. The features that are similar identify the presence of PMMA in all of them. The fingerprint characteristic vibration bands of PMMA appear at 1727 $v(\mathrm{C}=\mathrm{O})$ and $1450 v(\mathrm{C}-\mathrm{O})$. The bands at 3000 and $2900 \mathrm{~cm}^{-1}$ correspond to the $\mathrm{C}-\mathrm{H}$ stretching of the methyl group $\left(\mathrm{CH}_{3}\right)$ while the bands at 1300 and $1450 \mathrm{~cm}^{-1}$ are associated with $\mathrm{C}-\mathrm{H}$ symmetric and asymmetric stretching modes, respectively. The $1240 \mathrm{~cm}^{-1}$ band is assigned to torsion of the methylene group $\left(\mathrm{CH}_{2}\right)$ and the $1150 \mathrm{~cm}^{-1}$ band corresponds to vibration of the ester group $\mathrm{C}-\mathrm{O}$, while $\mathrm{C}-\mathrm{C}$ stretching bands are at 1000 and $800 \mathrm{~cm}^{-1}$. Absence of

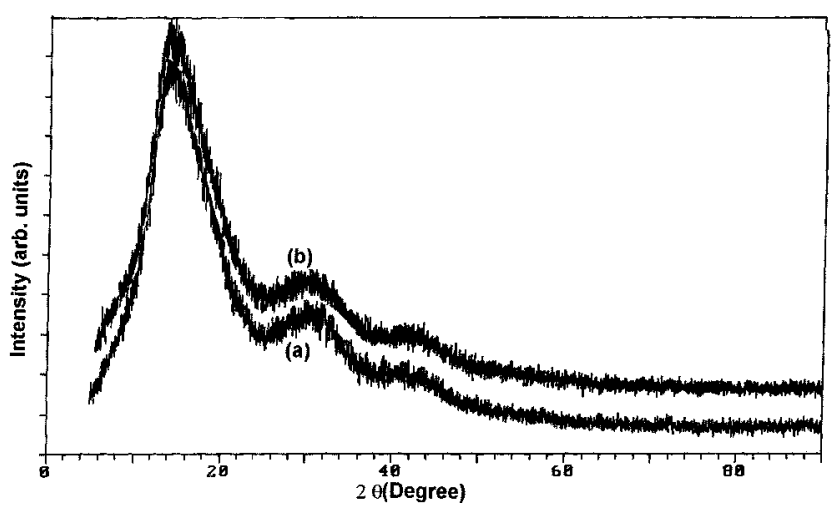

Figure 2. XRD pattern of (a) PMMA and (b) $\mathrm{PMMA}_{-} \mathrm{SiO}_{2}$ composites. any additional bands other than those of PMMA in the spectrum of PMMA and further they remaining unperturbed in all the three spectra indicate (i) the purity of the polymer obtained and (ii) formation of the nanocomposites.

The fingerprint frequencies of $\mathrm{SiO}_{2}$ are 464, 800, 950 and $1086.5 \mathrm{~cm}^{-1}$, respectively due to $\mathrm{Si}-\mathrm{O}-\mathrm{Si}$ bending, symmetric $\mathrm{Si}-\mathrm{O}-\mathrm{Si}$ stretching, $\mathrm{Si}-\mathrm{OH}$ flexible vibration and asymmetric $\mathrm{Si}-\mathrm{O}-\mathrm{Si}$ stretching vibration modes (Hwang et al 2005). In the spectrum of PMMA-SiO bands of PMMA also exist in the same frequency regions there are additional bands matching in their positions to above mentioned $\mathrm{SiO}_{2}$ modes (marked as $*$ ). The peak at $910-960 \mathrm{~cm}^{-1}$ is due to the overlapping from vibrations of Si-OH bonds (Jung and Park 2000).

In the high frequency region, peaks due to the stretching and in plane bending vibrations of the $\mathrm{OH}$ group of molecular $\mathrm{H}_{2} \mathrm{O}$ are observed at around 3400 and $1640 \mathrm{~cm}^{-1}$ in all the spectra. However, their intensity and sharpness differ. They are broad and weak in the PMMA spectrum, however, they are highly intense and sharp in the PMMA$\mathrm{TiO}_{2}$ spectrum. This latter feature can be explained due to superposition of the $v(\mathrm{OH})$ mode of interacting $\mathrm{H}$-bonds and the symmetric and antisymmetric $v(\mathrm{OH})$ mode of molecular water coordinated to $\mathrm{Ti}^{4+}$ cations. The signature band of $v(\mathrm{Ti}-\mathrm{O})$ vibration appears at $820 \mathrm{~cm}^{-1}$ (marked as *) which is very near to a band at $827 \mathrm{~cm}^{-1}$ due to $\mathrm{C}-\mathrm{C}$ stretching of PMMA.

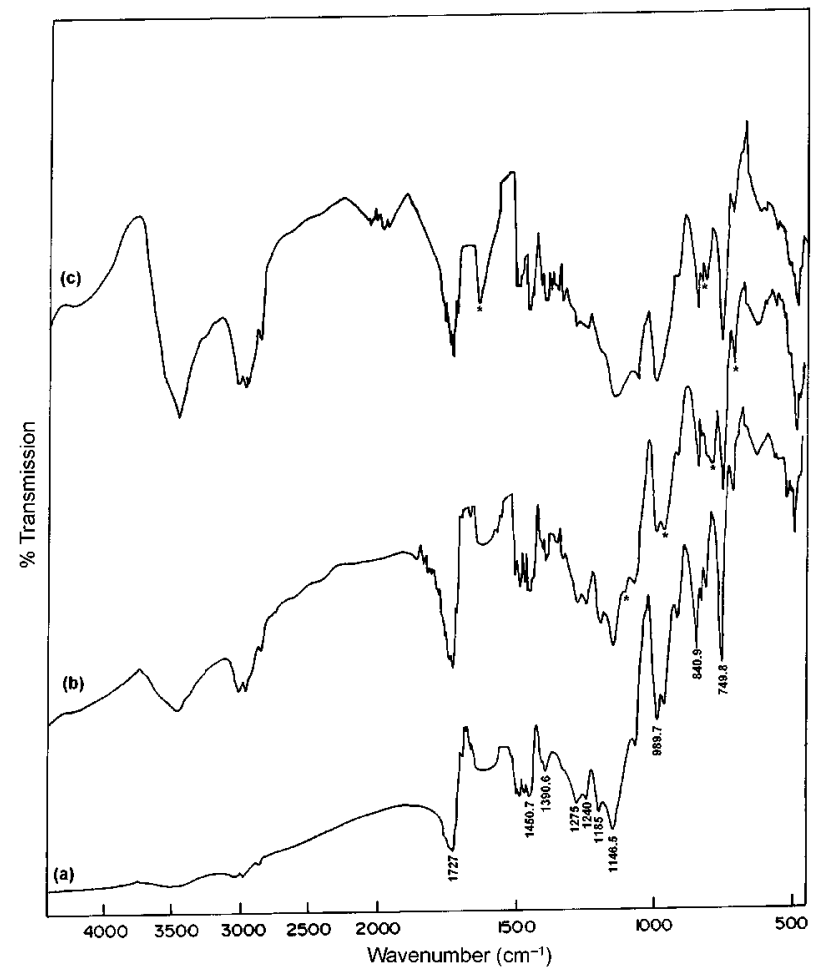

Figure 3. FTIR spectra of (a) PMMA, (b) PMMA-SiO ${ }_{2}$ and (c) PMMA-TiO . 


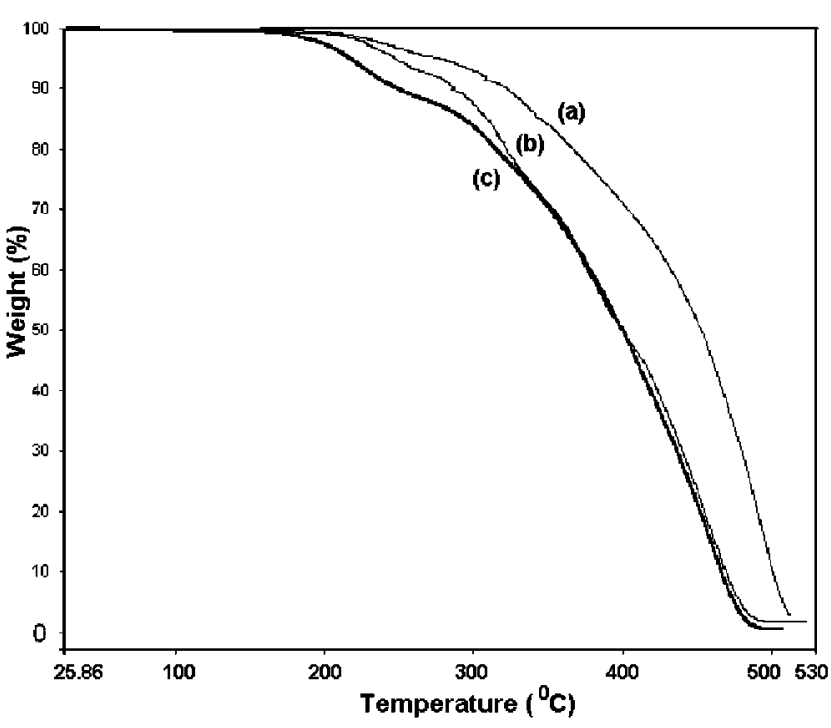

Figure 4. TGA curves of (a) PMMA, (b) $\mathrm{PMMA}^{-\mathrm{TiO}_{2}}$ and (c) PMMA-SiO ${ }_{2}$ composites.

\subsection{Thermal properties}

The thermal stability of these materials was investigated by TGA and the corresponding thermograms are illustrated in figure 4. The influence of nanocomposites on thermal behaviour was studied by comparing the degradation stability with the synthesized reference PMMA.

All the thermograms indicate no weight loss up to $200^{\circ} \mathrm{C}$ but a continuous weight loss with the same rate up to about $500^{\circ} \mathrm{C}$. The PMMA nanocomposites show slightly less thermal stability at $200^{\circ} \mathrm{C}$ probably due to physisorbed water evaporating at this temperature. Similar results have been reported by Kashiwagi et al (2003) for a nanocomposite synthesized by the addition of nanosilica particles into the polymer. In the thermograms the weight loss appears in two stages (i) $245-337 \cdot 5^{\circ} \mathrm{C}$ and (ii) $337 \cdot 5-420^{\circ} \mathrm{C}$. Both these reaction stages are seen to have comparable weight loss. The first one, which takes place by heat absorption, is ascribed to the degradation of the polymer's unsaturated groups, in contrast the second reaction is due to unzipping initiated by the random scission produced by monomer volatilization (Aymonier et al 2003). Beyond $425^{\circ} \mathrm{C}$ PMMA is destroyed completely. The TGA curves have been obtained under inert atmosphere i.e. in nitrogen, so they are identical, if obtained in air then increased thermal oxidative stability of the nanocomposites would have been evident. It is believed (Aymonier et al 2003) that the nanocomposites are more thermally stable in air than the reference polymer as it may shift upward the degradation temperature of the polymer.

\section{Conclusions}

An experimental protocol has been shown to synthesize PMMA based PMMA-SiO ${ }_{2}$ and $\mathrm{PMMA}^{-\mathrm{TiO}_{2}}$ nanocomposites. These nanocomposites were synthesized via sol-gel transformation in an in situ free radical polymerization of MMA. Morphological, thermal and structural studies have been carried out to characterize these nanocomposites. The SEM and FTIR studies support the formation of nanocomposite while XRD measurements reveal the amorphous nature of these nanocomposites. TGA investigations show that the formation of $\mathrm{SiO}_{2}$ and $\mathrm{TiO}_{2}$ nanoparticles did not alter the thermal degradation mechanism.

\section{Acknowledgements}

The work was supported by a grant from the Ministry of Non-Conventional Energy Source, India (Grant No. 15/8/ 2000-ST). One of us (SA) acknowledges CSIR for a senior research fellowship.

\section{References}

Ahmadi S J, Huang Y D and Li W 2004 J. Mater. Sci. 391919 Aymonier C, Bortzmeyer D, Thomann R and Mulhaupt R 2003 Chem. Mater. 154874

Burgos M and Langlet M 1999 J. Sol-Gel Sci. Technol. 16 267

Hwang S T, Hahn Y B, Nahm K S and Lee Y S 2005 Colloids Surf. A Physicochem. Eng. Aspects 25963

Jung K Y and Park S B 2000 Appl. Catal. B Environ. 25249

Kashiwagi T, Morgan A B, Antonucci J M, Van Landingham M R, Harris R H and Awad W H 2003 J. Appl. Polym. Sci. 89 2072

Lee L and Chen W C 2001 Chem. Mater. 131137

Limmer S J, Seraji S, Wu Y, Chou T P, Nguyen C and Cao G 2002 Adv. Funct. Mater. 1259

Mark J E 1996 Polym. Eng. Sci. 362905

Meneghetti P and Qutubuddin S 2004 Langmuir 203424

Meneghetti P, Qutubuddin S and Webber A 2004 Electrochim. Acta 494923

Nakajima A, Hashimoto K and Watanabe T 2001 Monatshefte für Chemie 13231

Qiang X, Chunfang Z, Zun Y J and Yuan C S 2004 J. Appl. Polym. Sci. 912739

Qutubuddin S and Fu X 2002 in Nano surface chemistry (ed.) M Rosoff (New York: Marcel Dekker Inc.) p. 653

Schmidt H 1988 J. Non-Cryst. Solids 10051

Suzuki N, Tomita Y and Kojima T 2002 Appl. Phys. Lett. 81 4121

Wang H W, Shieh C F, Chang K C and Chu H C 2005 J. Appl. Polym. Sci. 972175 